\title{
Production of Highly Sialylated Monoclonal Antibodies
}

\author{
Céline Raymond, Anna Robotham, John Kelly, Erika Lattová, \\ Hélène Perreault and Yves Durocher
}

Additional information is available at the end of the chapter

http://dx.doi.org/10.5772/51301

\section{Introduction}

The first monoclonal antibody (Mab), developed against kidney transplant rejection, was accepted by the FDA in 1986 [1]. Today, Mabs are leading the biotherapeutics market as 28 have been approved in Europe and the USA, and hundreds are in clinical trials [2-4]. Most of them are of IgG1 subtype, developed for cancer and immune disease treatments. Mabs clinical efficacy not only relies on specific target binding provided by their variable region, but also on their ability to trigger defense mechanisms such as antibody-dependent cellular cytotoxicity (ADCC) and complement dependent cytotoxicity (CDC). These effector functions are mediated by the interaction between the antibody Fc fragment and the Fc $\gamma$-receptors expressed on immune cell surfaces or the molecules of the complement involved in ADCC and CDC respectively. In the last decade, these interactions were found to be highly dependent on on the presence and structure of the N-glycan linked to the Fc fragment $[5,6]$.

Fc fragments possess two conserved N-glycosylation sites on asparagine 297 in the $\mathrm{C}_{\mathrm{H}} 2$ domain of each heavy chain. Mabs produced in mammalian cells possess a wide variety of glycoforms, as the attached glycans are modified to different extents with core-fucosylation, bisecting $\mathrm{N}$-acetylglucosamine addition, galactosylation and sialylation. The glycan composition is crucial, as the presence or absence of a single monosaccharide residue can remarkably affect the affinity of the Mab for the different Fc $\gamma$-receptors. Among the variety of monosaccharides present on Fc glycans, terminal sialic acids are particularly interesting, as their role in Mab functions is both positive and negative. Sialylation of the Fc glycan dramatically decreases Mab affinity for the canonical Fc receptors, thereby inhibiting ADCC. However, recent studies on the anti-inflammatory properties of intravenous immunoglobulins (IVIg) suggest that this biological activity could be conferred by the presence of $\alpha 2,6$-sialic acid residues on the Fc glycans. Although this hypothesis is still 
controversial, the study has raised a new interest in $\alpha 2,6$-sialylated IgGs. While glycosylation of therapeutic Mabs significantly impact their biological activity, the production of Mabs with a specific homogenous glycoform profile is in general beyond the reach of manufacturing bioprocesses. In this chapter, we describe and compare two large-scale transient expression platforms using chinese hamster ovary (CHO) and human embryonic kidney 293 (HEK293) cells for the production of highly sialylated monoclonal antibodies.

\section{IgG N-glycans and their interactions with the Fc}

Glycosylation is a complex process that involves several glycosyltransferases and glycosidases. Most glycosylation sites are located on the glycoprotein surface, whereas IgGs' $\mathrm{N}$-glycans are embedded within the Fc fragment. This particular location restricts the access of glycosyltransferases to their substrates, thereby reducing glycan complexity. Subsequently, while tri- and tetra-antennary glycans can be found on many glycoproteins such as EPO or IgG Fab fragments, Fc N-glycans are of the complex biantennary type [7] that consists of a heptasaccharide core structure comprising four N-acetylgalactosamines (GlcNAc) and three mannoses (Figure 1). The $\alpha 1,3$ and $\alpha 1,6$ arms can be further elongated with galactose and sialic acid. Fucose and bisecting-GlcNAc can be found on the core GlcNAc and on the central mannose respectively. This glycan is rarely fully processed; the predominant glycoform found on antibodies produced in $\mathrm{CHO}$ and 293 cell cultures is the fucosylated core-structure.

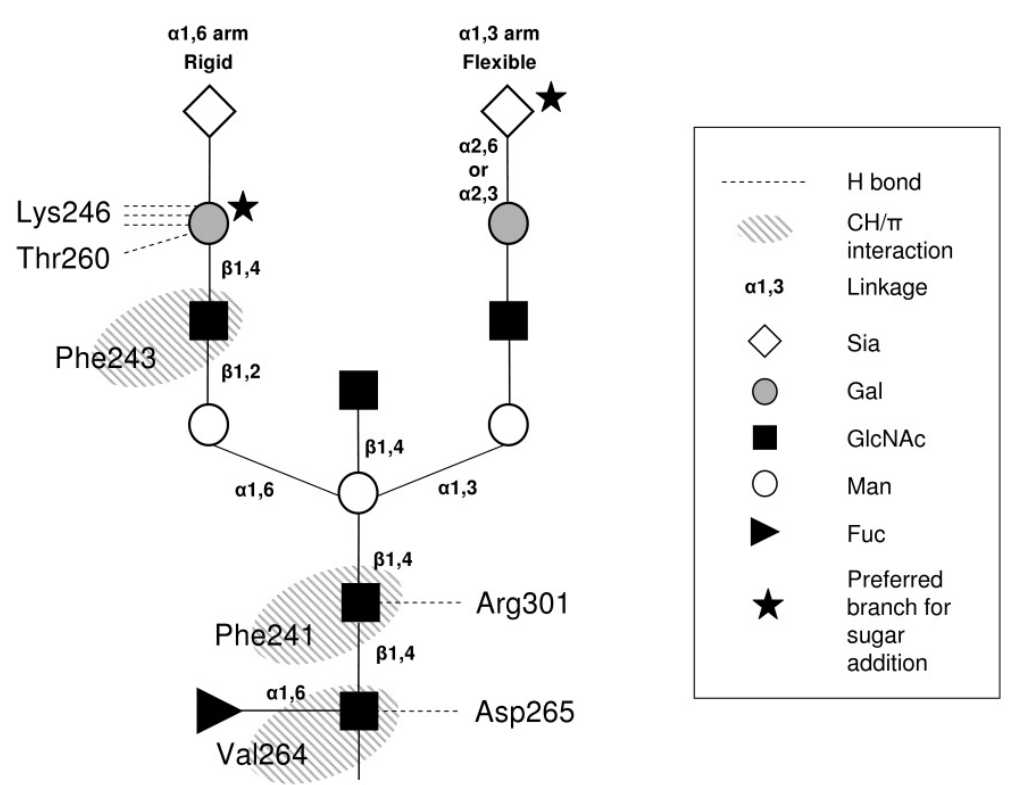

Figure 1. IgG1 glycan bi-antennary complex structure. Glycan interactions with $\mathrm{C}_{\mathrm{H}} 2$ amino acids. Sia: sialic acid; Gal: galactose; GlcNAc: N-acetylglucosamine; Man: mannose; Fuc: fucose. 
The $\mathrm{C}_{\mathrm{H}} 2$ amino acid sequence around Asn297 is very well conserved amongst IgG subtypes. Several amino acids have been shown to interact with the glycan located on the same heavy chain (HC), whereas no interaction is likely to happen with the other heavy chain. Amino acid-glycan interactions determine the glycan position within the Fc pocket and its availability for glycosyltransferases. The galactose (Gal) on the $\alpha 1,6 \mathrm{arm}$ is the main residue retaining the glycan on the protein surface $[6,8,9]$ by generating $\mathrm{H}$-bonds with Lys 246 and Thr260 [6]. In IgG1 and IgG4 subtypes, galactosylation occurs preferentially on $\alpha 1,6$ branch $[8,10]$. The inner saccharides have less interaction with the protein. The first and second GlcNAc residues generate H-bonds with Asp265 and Arg301 respectively [6]. They also form a CH/ $\pi$ interaction with the non-polar moieties of Val264 and Phe241 [6]. Similarly, the GlcNAc residue on the $\alpha 1,6$ branch also forms a $\mathrm{CH} / \pi$ interaction with Phe243 [11]. In contrast to galactosylation, sialylation preferentially occurs on the $\alpha 1,3 \mathrm{arm}$. It has been suggested that the galactose on the $\alpha 1,6$ arm may limit further elongation by maintaining the branch close to the $\mathrm{C}_{\mathrm{H}} 2$ protein surface [8]. However, even if steric hindrance may play a role, branch $\alpha 2$,6-sialylation specificity was shown to be a consequence of ST6Gal-I $\alpha 1,3$ arm preference [12], a phenomenon that is protein independent. The presence or absence of a sugar residue on the Fc glycan thus affects the conformation of the Fc, thereby affecting the Fc-mediated effector functions. These effects are summarized in Table 1.

\begin{tabular}{|c|c|c|c|c|}
\cline { 2 - 5 } \multicolumn{1}{c|}{} & $\begin{array}{c}\text { Mab-Fc } \gamma \mathrm{R} \\
\text { Affinity }\end{array}$ & $\begin{array}{c}\text { Mab-C1q } \\
\text { Affinity }\end{array}$ & Impacted effector function & Reference \\
\hline b-GlcNAc & + & & ADCC & Umaña 1999 \\
\hline Fucose & - & & ADCC & Shields 2002, Shinkawa 2003 \\
\hline Galactose & & + & CDC & Hodoniczky 2005 \\
\hline Sialic acid & - & & ADCC & Scallon 2007 \\
\hline
\end{tabular}

Table 1. Impact of the presence of extra-core monosaccharides on IgG ADCC and CDC effector functions.

\section{Sialic acids in Fc N-glycans}

The half-life of a number of glycoproteins can be enhanced by sialylation, as sialic acid acts as a cap that hides the penultimate galactose residue recognized by the hepatic asialoglycoprotein receptor (ASGPR, or Ashwell-Morell receptor) [13]. Sialic acid can be linked to the galactose either with $\alpha 2,3$ or $\alpha 2,6$ linkage. Recent studies showed that $\alpha 2,3$ sialylation provides a better protection to the protein than $\alpha 2,6$ sialylation, as ASGPR recognizes Sia $\alpha 2,6 \mathrm{Gal}$ and Sia $\alpha 2,6 \mathrm{GalNAc}$ moieties in addition to the well-known Gal and GalNAc residues [14, 15]. However, Fc-glycans have no apparent impact on IgG half-life [16]. The benefits of IgG sialylation on in vivo properties of IgGs are still to be understood. Recent studies suggest that sialylation provides anti-inflammatory properties to IgGs. It was observed that IVIg injected at very high doses have a therapeutic effect in several auto-immune and inflammatory diseases such as immune thrombocytopenic purpura 
(ITP) and rheumatoid arthritis (RA). Kaneko et al. demonstrated that IgGs bearing sialylated Fc-glycans have anti-inflammatory properties in a RA murine model [17]. The inhibitory $\mathrm{Fc}_{\mathrm{C}} \gamma$-receptor $\mathrm{Fc} \gamma \mathrm{RIIb}$ as well as Dendritic Cell-Specific Intercellular adhesion molecule-3-Grabbing Non-integrin (DC-SIGN) receptor were shown to be involved [1821], but the exact mechanism has not been elucidated. In parallel, Van de Geijn et al. reported that the increased levels of IgG1 galactosylation and sialylation during pregnancy may be responsible for the improved condition of RA-affected pregnant women [22].

IVIg are IgGs pooled and purified from sera of 3000 to 10000 donors. The sialylated fraction represents around $10 \%$ of the total IgGs present in the pool. To be therapeutically effective, IVIg used as an anti-inflammatory drug require repeated injections of very high doses (1$3 \mathrm{~g} / \mathrm{kg}$ ). As IVIg are successfully used in an increasing number of applications, a lack of donors is expected in the near future [23, 24]. To increase the therapeutic efficacy of antiinflammatory IgGs and avoid IVIg shortage, one strategy may reside in the production of recombinant sialylated IgGs [25].

\section{Production of sialylated recombinant antibody}

The $\mathrm{CHO}$ cell line is the most widely accepted production cell line in the industry for therapeutic manufacturing, including monoclonal antibodies. $\mathrm{CHO}$ cells have proven to be a safe expression system and provide high production yields. IgG and Fc fragment produced in $\mathrm{CHO}$ cells exhibit a very low level of sialylation $(<2 \%)$. However, it was shown that a single mutation in the $\mathrm{C}_{\mathrm{H}} 2$ domain, the replacement of Phe243 by an alanine, enhances the sialylation level of an IgG3 [26]. CHO glycosylation machinery is very similar to that found in human cells but with two major differences: they lack a functional $\mathrm{N}$ acetylglucosaminyltransferase-III (GnTIII) for the addition of bisecting-GlcNAc and, more importantly, they lack the alpha-2,6-sialyltransferase-I activity (ST6Gal-I or SIAT1) responsible for the addition of sialic acids on galactose residues with $\alpha 2,6$ an linkage [27]. The expression of a recombinant ST6Gal-I is therefore necessary to achieve the production of $\alpha 2,6$ sialylated IgGs in $\mathrm{CHO}$ cells.

Large-scale transfection strategies allow for the rapid expression of recombinant glycoproteins. The HEK293 cell line is easily transfectable with a variety of gene transfer reagents, and is probably the most utilized cell line for large-scale transient gene expression $[28,29]$. More recently, approaches using $\mathrm{CHO}$ cells have also been developed that provide tens to hundreds of milligrams of protein per litre [30]. In order to generate high titres of antibodies with enhanced $\alpha 2,6$-sialylation, we optimized the conditions for the transient coexpression of human ST6Gal-I together with IgG light and heavy chains in CHO and HEK293 cells. Our model antibody, Herceptin (or trastuzumab, herein abbreviated TZM), is a humanized mouse IgG1 used in the treatment of HER2-positive breast cancer. TZM has no glycosylation site on the Fab fragment but only the N297 sites on each $\mathrm{C}_{\mathrm{H}} 2$ domains of the Fc. We compared sialylation of the wild-type antibody to the F246A mutant (TZMm), which is equivalent to the IgG3 F243A mutant described previously [26]. 


\section{Materials and methods}

\section{Mammalian cell culture}

The human embryonic kidney 293-6E cell line stably expressing truncated Epstein-Barr virus Nuclear Antigen-1 (EBNA1) and the Chinese hamster ovary cell line also expressing a truncated EBNA1 protein (clone 3E7, or CHO-3E7) were grown in suspension culture in serum-free F17 medium (Invitrogen, Carlsbad, CA) supplemented with 0.1\% Pluronic-F68, $25 \mu \mathrm{g} / \mathrm{mL}$ geneticin G418 (for 293-6E cells only) and $4 \mathrm{mM}$ glutamine [31]. Cultures were maintained between 0.1 and $2.0 \times 10^{6}$ cells $/ \mathrm{mL}$ in $125 \mathrm{~mL}$ ventilated Erlenmeyer flasks shaken at $120 \mathrm{rpm}$ in a humidified incubator at $37^{\circ} \mathrm{C}$ with $5 \% \mathrm{CO}_{2}$.

\section{Plasmids}

The light (LC), heavy (HC), and F246A mutated heavy (HCF246A) chains were cloned between the EcoRI and BamHI sites of the pTT5 vector [32, 33]. The human ST6Gal1 gene was cloned between the HindIII and BamHI sites of the pYD7 vector [34]. Green fluorescent protein (GFP) cloned into the pTT vector was used as a reporter gene to evaluate transfection efficiency [35]. Plasmids were amplified in Escherichia coli (DH5 $\alpha$ ) grown overnight in CircleGrow medium (MP Biomedical, Solon, $\mathrm{OH}$ ) supplemented with 100ug/mL ampicillin and purified using MAXIprep or QIAprep spin Miniprep columns (Qiagen, Mississauga, ON).

\section{Transfection}

Linear 25 kDa polyethylenimine ${ }^{1}$ (LPEI), and linear deacylated polyethylenimine (PEI max) were obtained from Polysciences (Warrington, PA). Stock solutions $(1 \mathrm{mg} / \mathrm{mL}$ and $3 \mathrm{mg} / \mathrm{mL}$ for LPEI and PEImax respectively) were prepared in ultrapure water, sterilized by filtration $(0.2 \mu \mathrm{m})$, aliquoted and stored at $4^{\circ} \mathrm{C}$. Cells were diluted 2 days before transfection in fresh medium at 0.5 and $0.2 \times 10^{6}$ cells $/ \mathrm{mL}$ for $293-6 \mathrm{E}$ and $\mathrm{CHO} 3 \mathrm{E} 7$ cells respectively. Cells were transfected at densities between 1.5 and $2 \times 10^{6}$ cells $/ \mathrm{mL}$. DNA and PEI were separately diluted in complete serum-free F17 medium in sterile tubes. Transfection reagents volume was $10 \%$ of the final culture volume. The final DNA concentration was $1 \mu \mathrm{g}$ per $\mathrm{mL}$ of 293$6 \mathrm{E}$ culture, and $1.5 \mu \mathrm{g}$ per $\mathrm{mL}$ of $3 \mathrm{E} 7$ culture. PEI was used at the final concentration of $3 \mu \mathrm{g}$ per $\mathrm{mL}$ in 293-6E culture and $7 \mu \mathrm{g}$ per $\mathrm{mL}$ in 3E7 culture. Plasmid DNA mix was directly added to the cells and the suspension was allowed to incubate under agitation for $5 \mathrm{~min}$ at $37^{\circ} \mathrm{C}$ before the addition of PEI according to the direct transfection protocol [46]. Cells were fed 24 hours post-transfection (hpt) with TN1 peptone to a final concentration of $0.5 \%(\mathrm{w} / \mathrm{w})$ to enhance productivity [28]. Transfection efficiency was assessed $48 \mathrm{hpt}$ by determining the percentage of GFP positive cells and GFP fluorescence intensity by flow cytometry with a BD LSRII cytometer (BD Biosciences, Mississauga, ON). Only viable single cells were taken in account. Cell density and viability were determined using the Cedex Innovatis

\footnotetext{
1 "Use of PEI for transfection may be covered by existing intellectual property rights, including US Patent $6,013,240$, European Patent 0,770,140, and foreign equivalents for which further information may be obtained by contacting licensing@polyplus-transfection.com"
} 
automated cell counter Cedex Analyzer (Roche, Laval, QC) based on the trypan blue exclusion method.

Purification of Mabs from cell culture supernatants

Cell cultures were centrifuged $20 \mathrm{~min}$ at $3000 \mathrm{~g}$. The supernatant was collected and loaded on a $4 \mathrm{~mL}$ MabSelect SuRe column (GE Healthcare, Mississauga, ON) equilibrated in PBS. The column was washed with PBS and Mabs were eluted with $100 \mathrm{mM}$ citrate buffer $\mathrm{pH} 3.0$. The fractions containing Mabs were pooled and the citrate buffer was exchanged against water on Econo-Pac ${ }^{\circledR}$ 10DG columns (Bio-Rad, Mississauga, ON). Purified Mabs were sterilized by passing through $0.2 \mu \mathrm{m}$ filters, aliquoted, and stored at $-80^{\circ} \mathrm{C}$.

Quantification of Mabs

Concentration of TZM and TZMm in culture supernatants were determined by protein-A HPLC using a $800 \mu \mathrm{L}$ POROS ${ }^{\circledR} 20$ micron Protein A ID Cartridge (Applied BioSystems, Foster City, CA) according to the manufacturer's recommendations. The antibody present in the culture medium was also visualized following reducing and non-reducing SDS-PAGE stained by Bio-Safe Coomassie Stain (Bio-Rad, Mississauga, ON). Purified Mab were quantified by absorbance at $280 \mathrm{~nm}$ using a Nanodrop ${ }^{\mathrm{TM}}$ spectrophotometer (ThermoScientific).

\section{Lectin-Blot for $\alpha 2,6$-sialylation evaluation}

$\alpha 2,6$ sialylation was assessed by lectin-blotting on denatured and reduced antibody to separate HC from LC. After protein transfer, nitrocellulose membrane was incubated 3 hours with biotinylated Sambucus Nigra (SNA) lectin (Vector Laboratories, Burlingame, CA), then incubated with Streptavidin-Peroxidase Polymer (Sigma, Saint Louis, MO) for one hour. Signal was revealed with BM Chemiluminescence Blotting Substrate (POD) (Roche Applied Science, Indianapolis, IN).

Isoelectric focusing (IEF)

Purified Mabs were analysed on PhastGel ${ }^{\mathrm{TM}}$ 3-10, run on the PhastSystem ${ }^{\mathrm{TM}}$ (Amersham Biosciences, Baie d'Urfe, QC) according to the manufacturer's recommendations. Gels were fixed in trichloroacetic acid (TCA) $5 \%$ in water $(\mathrm{w} / \mathrm{w})$ and stained with Coomassie blue $0.02 \%$.

\section{Enzymatic removal of sialic acids}

$\alpha 2,3$ linked sialic acids were enzymatically removed from the purified antibody by Streptococcus pneumoniae Glyko Sialidase S (PROzyme, Hayward, CA) after one hour incubation at $37^{\circ} \mathrm{C}$. Total sialylation $(\alpha 2,6$ and $\alpha 2,3$ sialic acids) was removed after incubation overnight at $37^{\circ} \mathrm{C}$ with Arthrobacter ureafaciens Neuraminidase (MP Biomedicals, Solon, $\mathrm{OH})$.

\section{Mass spectrometry}

Peptide-N-glycosidase F (PNGaseF) was purchased from Roche (Mannheim, Germany). Phenylhydrazine (PHN), phenylhydrazine hydrochloride (PHN.HCl), iodomethane (MeI), 
dimethylsulfoxide (DMSO), 2,5-dihydroxybenzoic acid (DHB) and 2-aza-2-thiothymine (ATT) were obtained from Sigma (St. Louis, MO, USA). Carbon and STRATA-X-C cartridges were purchased from Phenomenex (Torrance, CA). Solid sodium hydroxide $(\mathrm{NaOH})$ and HPLC-grade solvents (acetonitrile (ACN), chloroform, methanol) were obtained from Fisher Scientific (Fair Lawn, NJ). HPLC-grade water was obtained with a Milli-Q® plus TOC water purification system (Millipore, Bedford, MA).

\section{Release of N-linked oligosaccharides from samples and MALDI-MS analysis}

To the sample solution (100 $\mu \mathrm{L} ; 50 \mu$ g glycoprotein) $2 \mu \mathrm{L}$ of PNGaseF were added and incubated at $37^{\circ} \mathrm{C}$ for $5-18 \mathrm{~h}$. One $\mu \mathrm{L}$ of digested mixture was loaded onto a spot with freshly prepared matrix solution $(0.8 \mu \mathrm{L} ; 5 \mathrm{mg}$ of ATT and $2.5 \mathrm{mg}$ of $\mathrm{PHN} . \mathrm{HCl}$ dissolved in $350 \mu \mathrm{L} 50 \% \mathrm{ACN}$ in water) predeposited on the surface of a MALDI target. When the mixture was partially dried, $0.4 \mu \mathrm{L}$ of PHN-labelling solution (phenylhydrazine:deionized water:ACN/1:4:1) was added to the spot with sample-matrix and left to air air-dry. The samples were analysed by MALDI-TOF/TOF-MS (UltrafleXtremeTM, Bruker) in both positive and negative ion modes. For the structural analysis individual parent ions were manually selected. MS/MS spectra of oligosaccharides were interpreted manually. The structures of N-glycans were derived on the basis of fragmentation patterns which were produced under MS/MS conditions for single precursor ions. For a discrimination of isomeric structures, general rules described in previous study have been applied [36].

To verify glycan profiles obtained by PHN-target derivatization immediately after PNGaseF treatment, all digested samples were also purified on STRATA-XC cartridges combined with carbon cartridges. After washing carbon cartridge with $4 \times 1000 \mu \mathrm{L}$ of deionized water, glycans were eluted with $40 \%$ ACN $(1000 \mu \mathrm{L})$. After total evaporation the glycans were labelled with PHN as described above or permethylated according to the procedure described by Ciucanu and Kerek [47]. Briefly, dried fractions containing oligosaccharides were dissolved in DMSO $(40 \mu \mathrm{L})$, to which $\mathrm{NaOH}(2 \mathrm{mg})$ and methyl iodide $(8 \mu \mathrm{L})$ were added. The mixture was vortexed vigorously $(30 \mathrm{~min})$ at room temperature. Then, the reaction was stopped by adding ice-cold water $(500 \mu \mathrm{L})$ followed by chloroform $(200 \mu \mathrm{L})$. After mixing and centrifugation, the upper aqueous layer was discarded and the chloroform portion was again washed with distilled water $(4 \times 500 \mu \mathrm{L}$ each). Chloroform was evaporated and the sample was reconstituted in $70 \%$ aqueous methanol $(5 \mu \mathrm{L})$.One $\mu \mathrm{L}$ of permethylated sample solution was spotted into DHB matrix and analysed by MS.

\section{Intact mass analysis of Mabs}

The antibody isolates were analysed by Trap-LC-MS using an Agilent 1100 series HPLC system coupled to a Q-TOF2 hybrid quadrupole time-of-flight mass spectrometer (Waters) equipped with an electrospray interface. Approximately $3 \mu \mathrm{g}$ of antibody was injected onto a Protein MicroTrap (Michrom BioResources/Dionex). The trap was washed with deionized water $(\sim 500 \mu \mathrm{L})$ to remove buffers prior to being switched on-line with the LC-MS system. The antibody was eluted using a linear gradient from $1 \%$ to $85 \%$ solvent $\mathrm{B}$ in $27 \mathrm{~min}$ (solvent A: $0.1 \%$ formic acid, solvent B: $0.1 \%$ formic acid in acetonitrile, flow rate: $0.45 \mathrm{~mL} / \mathrm{min}$ ). The mobile phase was split before the trap to $50 \mu \mathrm{L} / \mathrm{min}$. The electrospray voltage was $4200 \mathrm{~V}$ 
and cone voltage was $80 \mathrm{~V}$. The Q-TOF2 mass spectrometer was calibrated for high mass range using cesium iodide. Mass spectra were acquired every 2 seconds during the analyses over the full mass range ( $\mathrm{m} / \mathrm{z} 50-4000)$. The mass spectra acquired across the protein peak were combined and smoothed (smooth window: \pm 3 , number of smooths: 8 , smoothing method: Savitsky-Golay) and deconvoluted using the MassLynx MaxEnt 1 software from Waters: spectral window: m/z 2500-3200, molecular weight range: 140,000-160,000 Da, resolution: $1.00 \mathrm{Da} /$ channel, damage model: simulated isotope pattern spectrometer blur width of $0.500 \mathrm{Da}$, minimum intensity ratios: $60 \%$ left, $60 \%$ right, number of iterations: 15 ). Peaks in the molecular weight profile were integrated using the MassLynx software.

\section{Results}

\subsection{Transfection conditions optimization}

\section{DNA ratio parameters}

Transfection efficacy relies on a variety of parameters that are, amongst others, cell lineprotein- and plasmid-dependent. We expressed our model Mab together with the human $\alpha 2,6$ sialyltransferase (ST6Gal-I) by performing co-transfection of the Mab heavy chain (HC), light chain (LC) and ST6Gal-I expressing plasmids. The optimal proportions of each plasmid were determined to ensure 1) the proper assembly of the Mab, 2) high sialylation levels and 3) high Mab yields. The LC:HC and (LC:HC):ST6Gal-I ratios were then assessed in CHO and 293 cells in suspension in 6-well plates, after 5 days at $37^{\circ} \mathrm{C}$. The supernatants were collected and analysed by protein-A HPLC (Fig. 2A) and by SDS-PAGE followed by Coomassie staining (Fig. 2B) to evaluate the expression yield and proper assembly of antibodies. Sialylation was assessed by SNA-blotting (Fig. 2C). For TZMm, a slight excess of LC plasmid (LC:HC of 6:4) provided a better expression yield in both cell lines. For wild-type TZM, this ratio was of 5:5 in 293 and 6:4 in CHO cells. A ratio 6:4 was chosen for the expression of TZM and TZMm in both cell lines. As for sialylation, 20\% of ST6Gal-I plasmid was found to allow maximum signal in SNA blots without significantly affecting TZMm expression yields in both cell lines. It is worth noticing that the sialylation of the wild-type antibody remained very low for all ST6Gal-I plasmid ratios, suggesting the inability of the enzyme to sialylate TZM. Mab yields were increased in 293 cells with the incorporation of $30 \%$ of non-coding DNA (salmon sperm DNA) in the final DNA mix, while this was unnecessary in CHO cells (data not shown).

\section{Transfection protocol}

The usual polyethylenimine (PEI) mediated transfection protocol involves the formation of DNA-PEI complexes (polyplexes) prior to their addition to the cells. Another approach, the direct transfection, consists of the successive addition of DNA and PEI to the cell suspension, and was validated with 293 cells to facilitate large-scale transfection handling $[46,48]$. This strategy was adapted here for CHO cells. $1.5 \mu \mathrm{g}$ of DNA and $7 \mu \mathrm{g}$ of PEI per $\mathrm{mL}$ of culture allowed the expression of amounts of IgGs equal to the classic indirect protocol (Fig. 3). Transfection parameters for $\mathrm{CHO}$ and 293 cells are summarized in Table 2. 
A

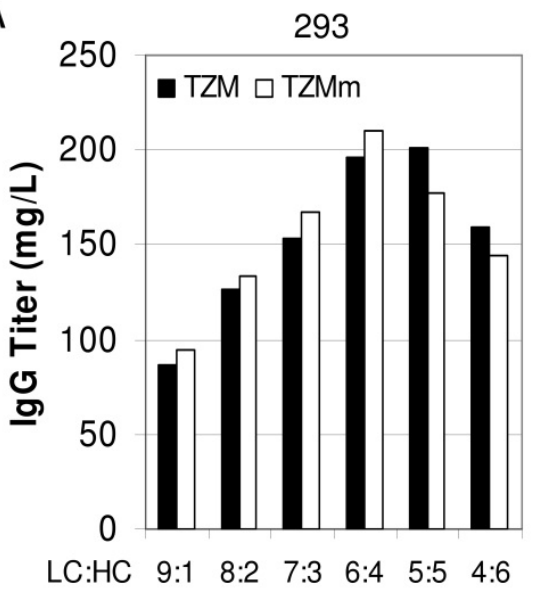

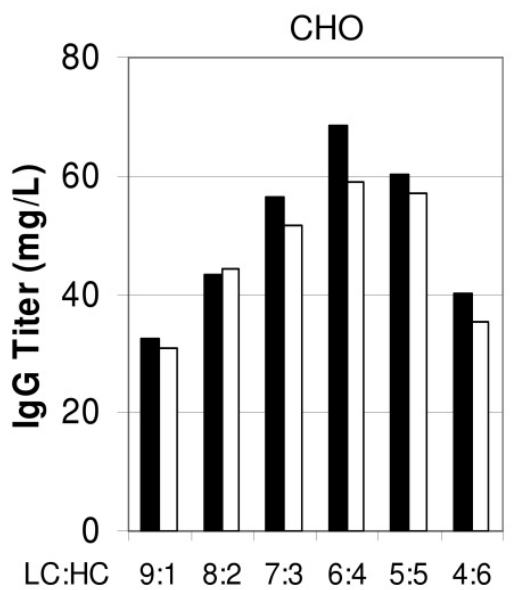

B

$\begin{array}{llllllllllllllllllllllll}1 & 2 & 3 & 4 & 5 & 6 & 1 & 2 & 3 & 4 & 5 & 6 & 1 & 2 & 3 & 4 & 5 & 6 & 1 & 2 & 3 & 4 & 5 & 6\end{array}$
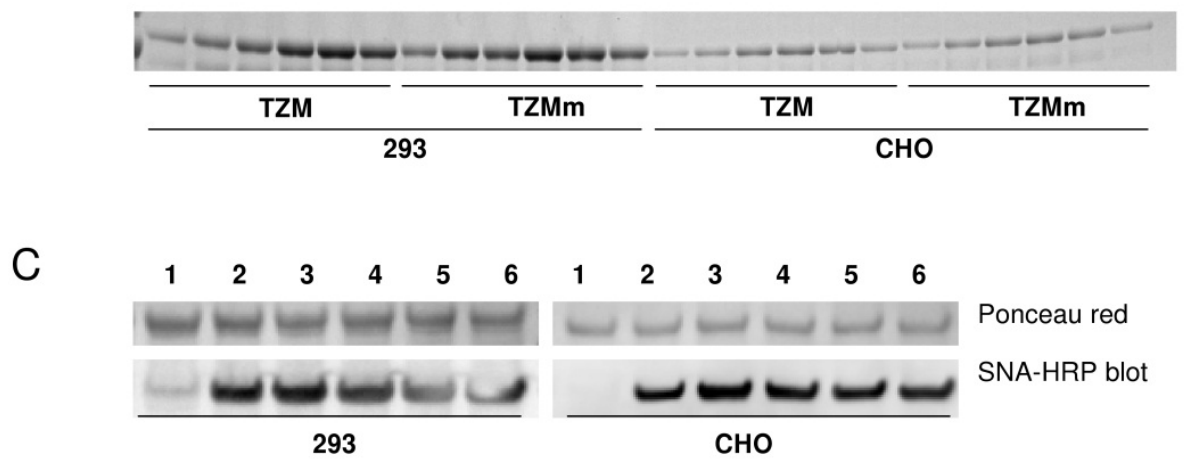

Figure 2. Optimization of LC:HC and ST6Gal-I ratio. Panel A - Mabs were quantified by protein-A HPLC in 293 and CHO supernatants 5 days post-transfection. Different LC:HC and LC:HC F246A DNA ratios were tested for proper expression of TZM and TZMm respectively. Panel B - The supernatants were loaded on SDS-PAGE (non-reducing) and stained with Coomassie blue. LC:HC ratios are: Lane 1: 9:1; Lane 2: 8:2; Lane 3: 7:3; Lane 4: 6:4; Lane 5: 5:5; Lane 6: 4:6. Panel C - Determination of the optimal ST6Gal-I ratio. Supernatants from cells transfected with TZMm HC and LC and various ST6Gal-I ratios were harvested 5 days post-transfection, separated by SDS-PAGE (reducing conditions) and transferred on nitrocellulose membrane for the determination of the sialylation level by blotting with SNA-HRP. Nitrocellulose membrane was stained with Ponceau red for protein load control, or incubated with SNA-HRP. Proportion of ST6Gal-I plasmid in transfection mixture was: Lane 1: 0\%; Lane 2: 10\%; Lane 3: 20\%; Lane 4: 30\%; Lane 5: 40\%; Lane 6: 50\%. 


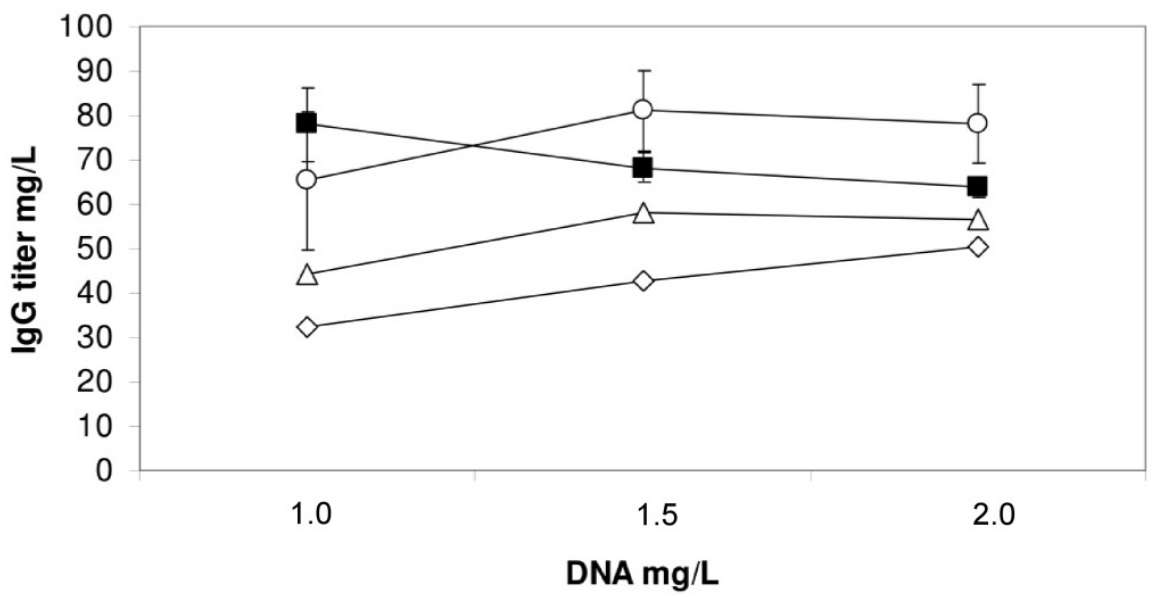

Figure 3. Determination of the optimal transfection parameters. Final concentrations of DNA and PEI per $\mathrm{mL}$ of culture for direct transfection of $\mathrm{CHO}$ cells were determined for TZM. Closed squares represent the TZM titres obtained using the traditional indirect protocol, i.e. with DNA-PEI complexation prior to the addition to the cell culture (PEI $5 \mu \mathrm{g} / \mathrm{mL}$ ). Open symbols represent the results for 3 different PEI concentrations with direct protocol: open circle: $7 \mu \mathrm{g} / \mathrm{mL}$; open triangle: $6 \mu \mathrm{g} / \mathrm{mL}$; open diamond $5 \mu \mathrm{g} / \mathrm{mL}$. Results represent the average of three independent experiments $\pm \mathrm{SD}$.

\begin{tabular}{|lll|}
\hline & 293 & CHO \\
\hline LC:HC & $6: 4$ & $6: 4$ \\
\hline (LC:HC):ST6Gal-I & $(80): 20$ & $(80): 20$ \\
\hline$(($ LC:HC):ST6Gal-I):ssDNA & $(70): 30$ & $(100): 0$ \\
\hline DNA:PEI for Direct protocol & $1: 3$ (LPEI) & $1.5: 7$ (PEI max) \\
\hline
\end{tabular}

Table 2. Optimal DNA and PEI ratios determined for the expression of TZM and TZM F246A mutant (TZMm) in 293-6E and CHO cells using the direct transfection protocol.

\subsection{Mab expression and sialylation kinetics}

Sialylation is likely to be limited after a certain time in batch culture due to a combination of adverse factors such as, but not limited to, ammonia accumulation [37, 38], ST6Gal-I plasmid loss upon successive cell division and sialidases release in medium [39]. As Mab titre increases over time in the culture medium, the harvest time point selected must provide an acceptable compromise between high Mab yield and high sialylation. In this study, we gave priority to sialylation over Mab expression yield.

Sialylation kinetics was studied on the mutant antibody as sialylation of TZM was barely detectable. TZMm co-expression with ST6Gal-I was carried out for 7 days in 293 and CHO cells, until viability dropped to approximately $60 \%$ (Fig. 4A). A fraction of each culture was harvested daily from day 3 to day 7 and purified by protein-A affinity chromatography. Sialylation level of the purified TZMm fractions were assessed by SNA-blotting and IEF analyses. 
A

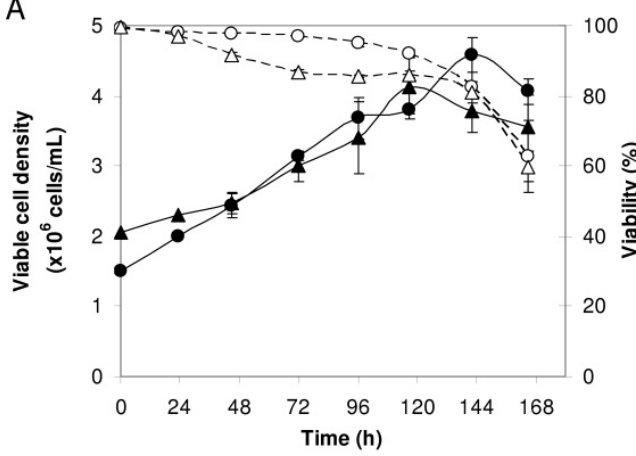

B

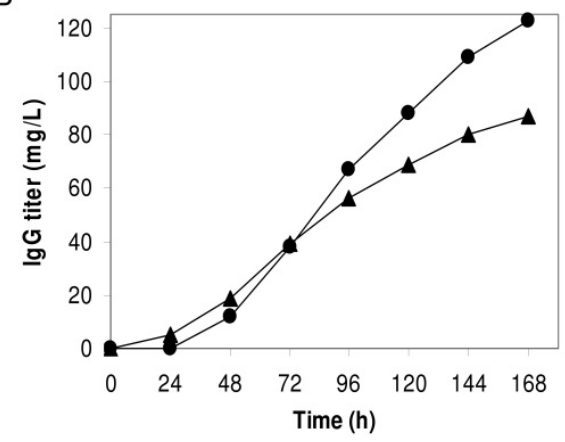

Figure 4. Production kinetic of TZMm in 293 and CHO cells. Panel A - Cell growth and viability for TZMm co-expression with ST6Gal-I. Cells were transfected at $\mathrm{t}=0 \mathrm{~h}$ and fed at $24 \mathrm{~h}$ post-transfection with TN1 peptone $(0.5 \% \mathrm{w} / \mathrm{v}$ final). Open and closed circles: viability and viable cell density in 293 cell cultures. Open and closed triangles: viability and viable cell density in $\mathrm{CHO}$ cell cultures. Panel B TZMm yield over time as determined by protein-A HPLC quantification of each fraction. Closed circles: titres in 293 cells; closed triangles: titres in CHO cells.

In 293 cells, SNA-blot showed a maximum $\alpha 2,6$-sialylation on day 3, which then decreased over time (Fig. 5A). In CHO cells, no significant loss of intensity was observed, suggesting that $\alpha 2,6$-sialylation of the heavy chain was more stable over time. These patterns were supported by IEF analyses. Due to the negative charge of sialic acid, mono, di, tri and tetrasialylated Mabs are expected to have lower pI than asialylated glycoforms. Even though these variations in $\mathrm{pI}$ are small, they could be easily distinguished on IEF gels. The upper band intensity showed a higher amount of asialylated glycoforms in 293 than in CHO as seen on the blot (Fig. 5B). The decline in the sialylation level was less obvious by IEF vs SNA-blot analysis for the 293 cells, which could be explained by a change in the $\alpha 2,3 / \alpha 2,6$ sialylation ratio over time. For $\mathrm{CHO}$ cells, the IEF profiles mirrored the SNA-blot results as there was no significant band intensity variation among the samples from day 3 to day 7 . TZM and TZMm treated overnight with $\alpha 2,3-6$ neuraminidase (Arthrobacter ureafaciens) at $37^{\circ} \mathrm{C}$ exhibited the same pattern than aglycosylated TZM mutant N297A (Fig. 5C), confirming that the lower bands correspond to sialylated states of the Mabs. 
A

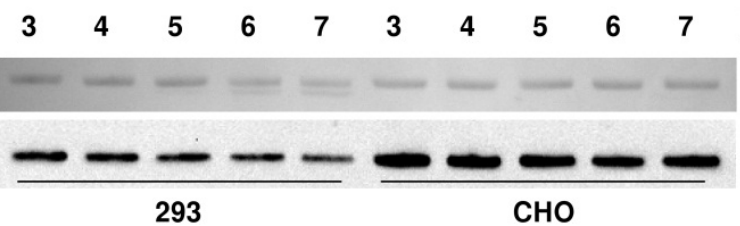

Days post-transfection

Ponceau red

SNA-HRP blot

293

CHO

B

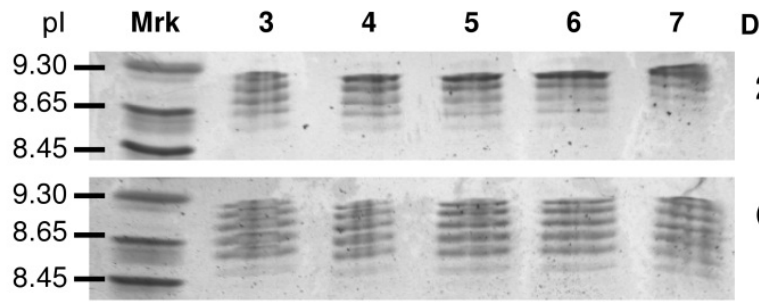

Days post-transfection

293

$\mathrm{CHO}$

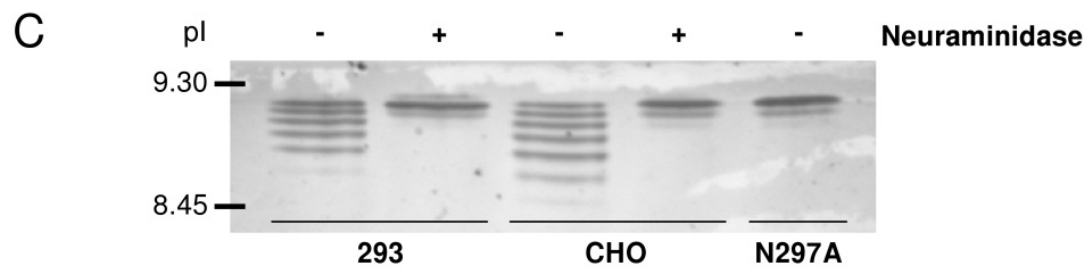

Figure 5. Sialylation kinetic of TZMm in 293 and CHO cells. Panel A -TZMm was co-expressed with ST6Gal-I, harvested from day 3 to day 7 and purified by protein-A chromatography. 300ng were resolved by SDS-PAGE (reducing condition), transferred to nitrocellulose membrane and probed with SNA-HRP. Only the heavy chain is shown. The membrane was stained with Ponceau red to monitor protein loads. Panel B - IEF profiles of purified TZMm co-expressed with ST6Gal-I and harvested between day 3 and 7 post-transfection. Mrk: IEF calibration markers. Panel C - IEF profiles of purified TZMm from 293 and $\mathrm{CHO}$ cells digested or not with A. ureafaciens neuraminidase and of aglycosylated TZM (N297A mutant produced in 293 cells).

\subsection{Impact of ST6Gal-I expression and F246A mutation on sialylation patterns}

\section{Impact of ST6Gal-I overexpression and F246A mutation on TZM sialylation}

To evaluate the impact of ST6Gal-I expression on overall sialylation levels of TZM and TZMm, purified Mabs were submitted to SNA blotting and IEF analyses (Figure 6). While SNA blot (panel A) shows a significant increase in $\alpha 2,6$-sialylation levels of TZMm, no increase in sialylation of the wild-type antibody could be observed in 293 cells while only a faint band could be seen in CHO cells. In 293 cells, only a marginal signal with SNA lectin could be seen when TZMm was expressed alone, suggesting that endogenous levels of ST6Gal-I activity are low in this cell line. The main consequence of ST6Gal-I co-expression 
was the significant preference for $\alpha 2,6$-sialylation over $\alpha 2,3$-sialylation of TZMm in CHO cells. While all of the acidic TZMm species observed when the Mab was expressed alone disappeared after treatment with Sialidase $S$ (an $\alpha 2,3$-specific neuraminidase), these acidic species became resistant to Sialidase S when ST6Gal-I was co-expressed. This suggests that sialylation of TZMm in the presence of ST6Gal-I was mostly due to the preferential addition of $\alpha 2,6$ - over $\alpha 2,3$-sialic acid residues.

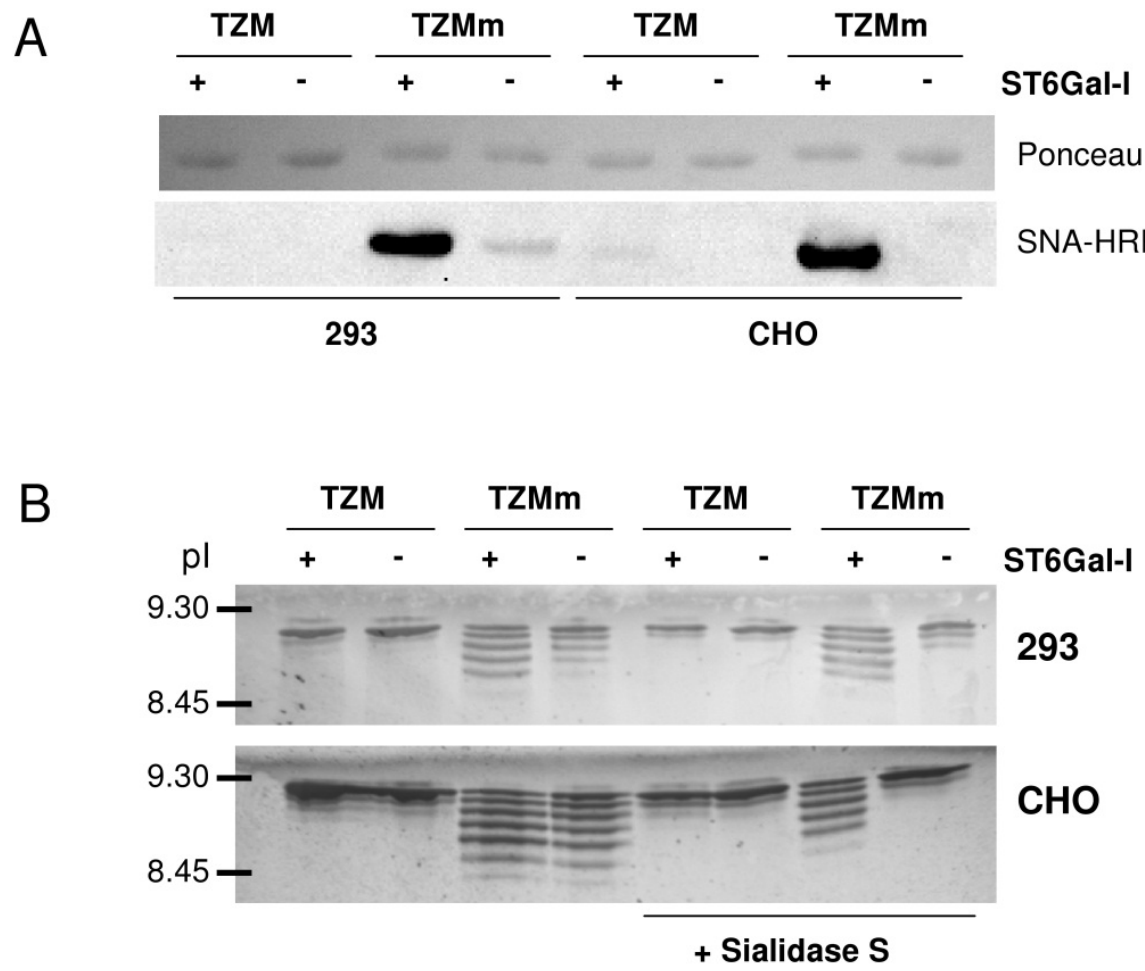

Figure 6. Impact of ST6Gal-I expression and F246A mutation on TZM sialylation. Transfected CHO and 293 cultures (with or without ST6Gal-I) were harvested on day 3 and day 4, respectively and Mabs were purified by protein A chromatography. Panel A - Mabs (300ng per lane) were resolved by SDS-PAGE (reducing conditions) and submitted to SNA-blotting (only the heavy chains are shown). Panel B - IEF analyses of Mabs from 293 and CHO cells treated or not with Sialidase S ( $\alpha 2,3$-neuraminidase).

Glycan analysis by MALDI-MS spectrometry

After PNGaseF digestion of the purified Mabs, oligosaccharide detection was firstly achieved by MALDI-MS directly after non-reductive on-target derivatization with phenylhydrazine (PHN) without using any purification procedure. Despite a presence of side products and salts coming from original buffer, which generally have a tendency to suppress signal peaks corresponding to glycans, all samples provided good evidence for the presence of N-glycans. 
These N-glycans were known to be located on the Fc fragment as Asparagine 297 is the only glycosylation site in our model antibody. The profile differences between the samples in $\mathrm{CHO}$ and 293 were consistent with what was observed by SNA-blot and IEF. The dominant oligosaccharides derived from all Mab samples were observed in positive mode in PHNderivatized samples at $\mathrm{m} / \mathrm{z}$ 1575, 1737 and were consistent with biantennary core-fucosylated structures with zero and one galactose. Glycan having both antennae occupied with galactoses $(\mathrm{m} / \mathrm{z}$ 1899) was observed with higher intensity only in TZMm samples without ST6Gal-I.

The spectra recorded in the negative mode from TZM samples co-expressed with ST6Gal-I and derivatized with PHN, provided evidence for the presence of small amount of

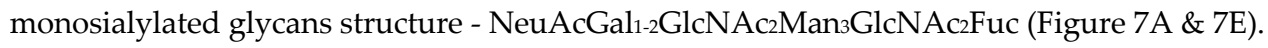

Sialylated glycans were clearly detected at higher levels in TZMm samples. TZMm samples co-expressed with ST6Gal-I in both cell lines (Fig. 7C \& 7G) indicated higher sialylation degree than that obtained under control conditions (Fig. 7D \& 7H).
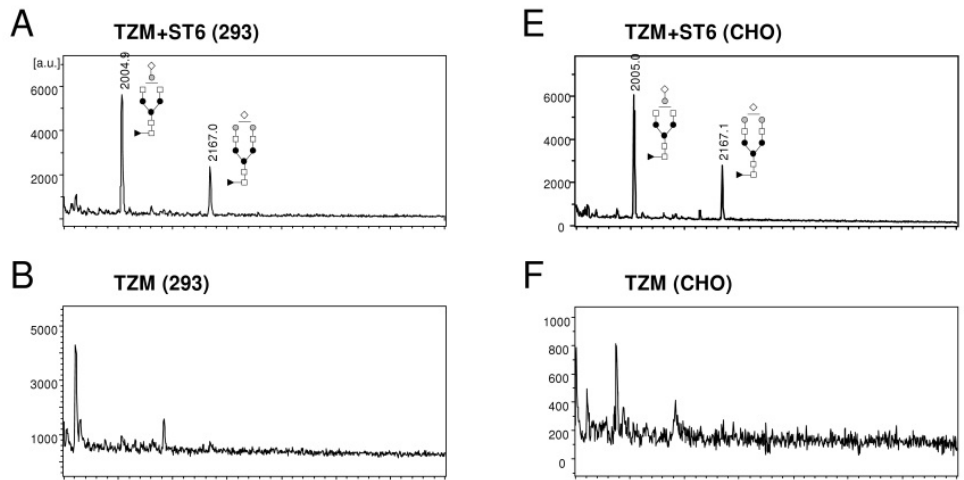

$\mathrm{F}$
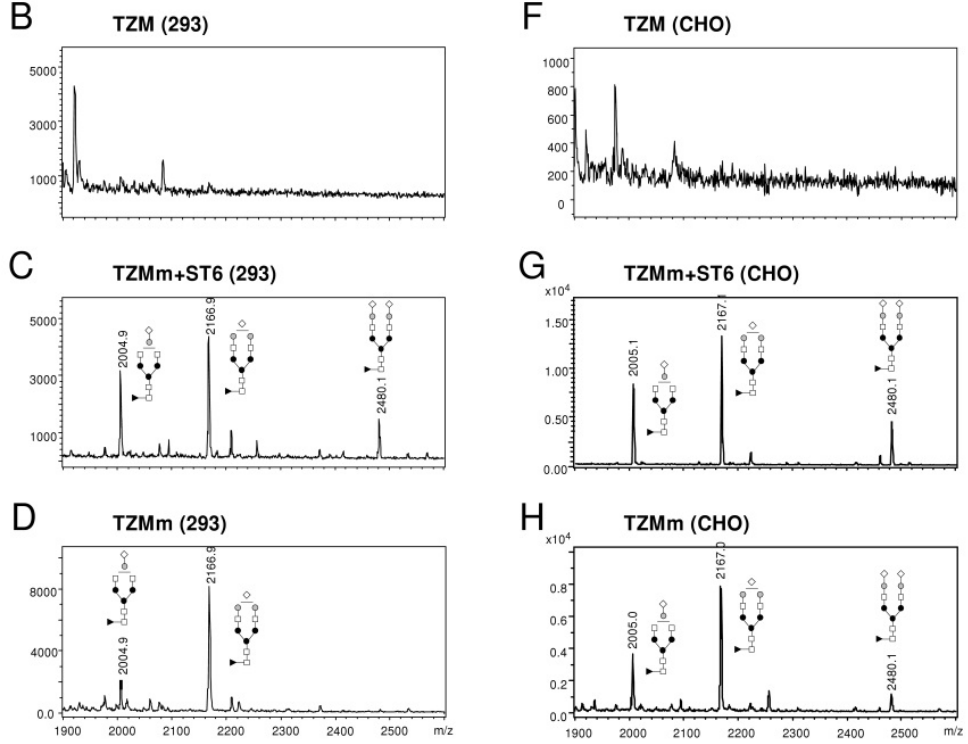

Figure 7. Glycan analysis by MALDI-MS in negative mode. Negative MALDI-MS spectra of N-Glycans obtained after PNGase F digestion and on-target derivatization with PHN (+90 Da) of MAbs from 293 cells (panels A to D) and CHO cells (panels E to H). Monosialylated glycans (m/z at 2005 and 2167) are detected as $[\mathrm{M}-\mathrm{H}]^{-}$ions. 
The high level of sialylated glycans was even better observed in the fractions obtained after purification prior to derivatization, as shown in Figure 8 which represents MS spectra recorded from TZMm samples produced in 293 cells. Interestingly, this analysis revealed traces of triantennary glycans in TZMm that cannot naturally occur in wild-type IgG1, supporting the vision of an open Fc conformation presenting reduced space constraint to the glycosyltransferases.
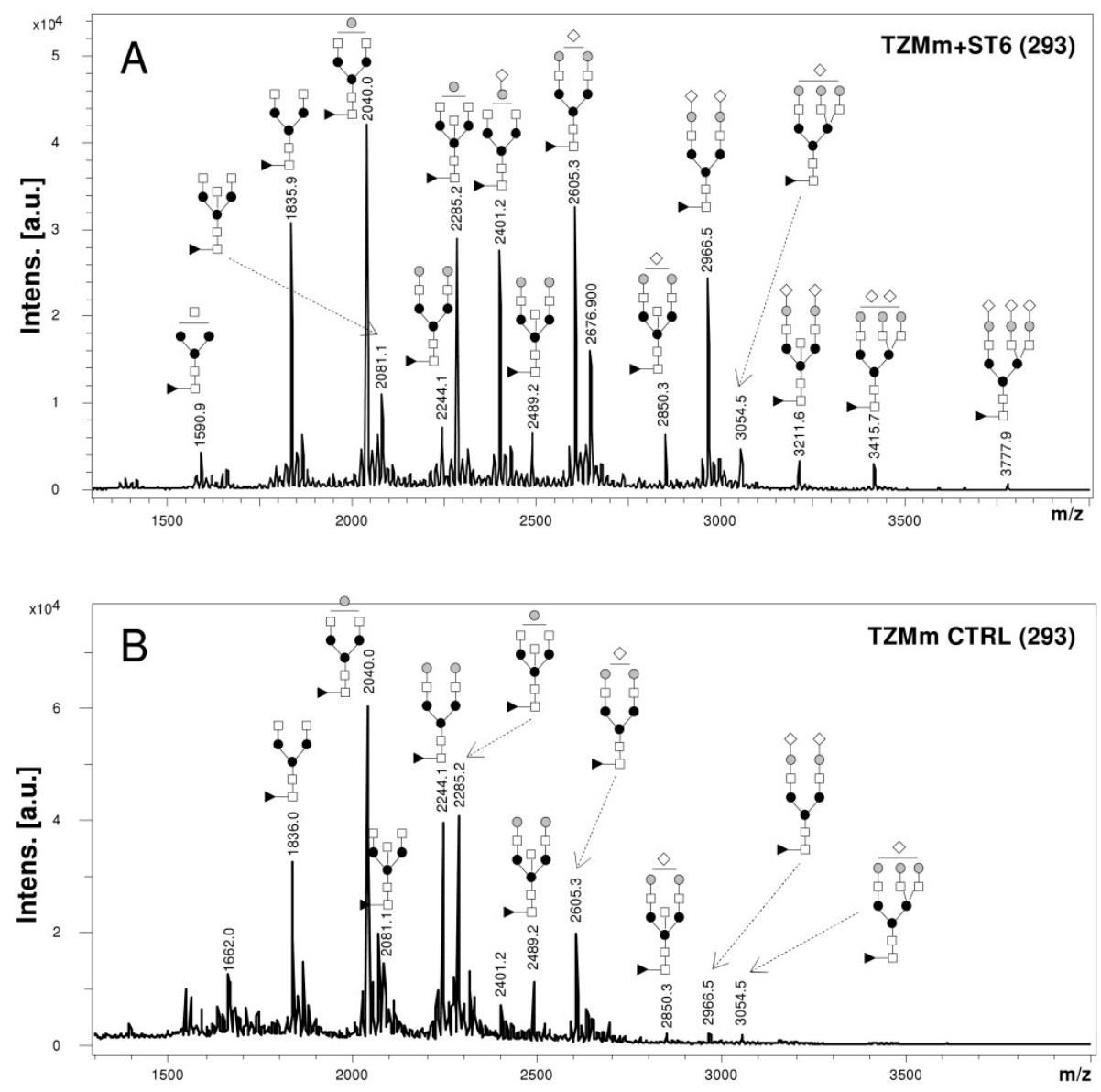

Figure 8. Glycan analysis by MALDI-MS in positive mode. Positive MALDI-MS spectra of N-glycans recorded after glycans purification and permethylation from PNGase digested TZMm produced in 293 cells. All glycans are as $[\mathrm{M}+\mathrm{Na}]^{+}$. 
Intact mass analysis of antibodies by LC-ESI-MS using a Q-TOF2 spectrometer

The intact mass analysis approach allowed us to see the outcome of the pairing of the glycans on the overall glycosylation of the antibodies, and to distinguish with a high resolution between galactosylated and sialylated Mabs glycoforms. The prevalence of agalactosylated and monogalactosylated glycoforms in TZM+/-ST6 samples in both cell lines was consistent with the glycan analysis by MALDI-MS (Fig. 9A). No trace of sialylated

A

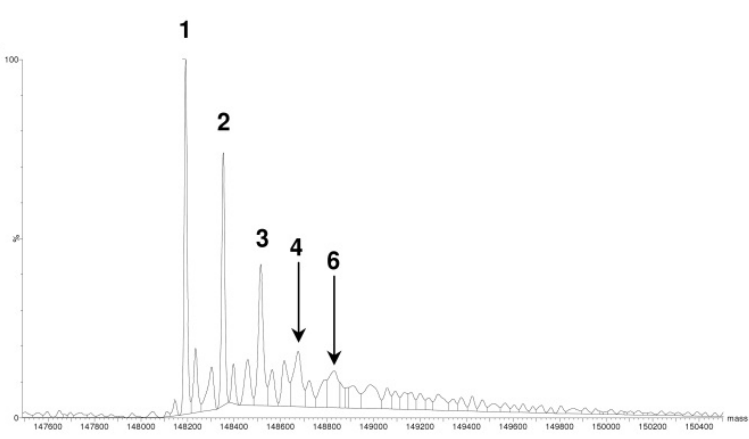

B

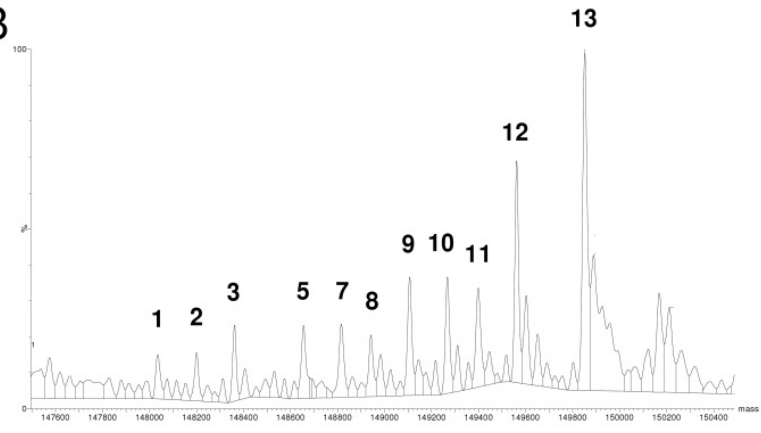

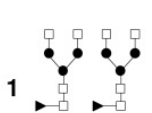
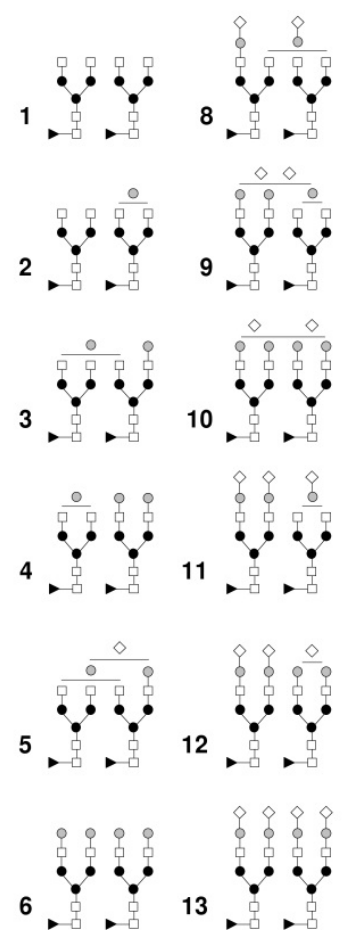

13

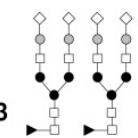

Figure 9. Intact antibody analysis of Mabs by by LC-ESI-MS. Purified Mabs produced in CHO cells were analyzed by LC-ESI-MS on a Q-TOF2 spectrometer as described in Materials and Methods. Panel A: TZM+ST6; panel B: TZMm+ST6. 
antibodies was detected. The close resemblance of the profiles of TZM produced in the presence or absence of ST6Gal-I confirmed the poor sialylation efficacy of the wild-type antibody. In contrast, the association of the Fc F246A mutation with the expression of ST6Gal-I in CHO cells resulted in a massive conversion of the neutral Mabs into a variety of mono-, di-, tri- and tetrasialylated antibodies, where the tetrasialylated version was predominant (Fig. 9B). The combination of the two approaches in 293 cells, as well as the FC mutation alone in both cell lines provided intermediate results (not shown).

\section{Discussion}

IVIg are successfully used for the treatment of an increasing number of autoimmune and inflammatory disorders. Their efficacy results from several distinct mechanisms working together in a complex fashion that is not yet understood. Anti-inflammatory mechanisms of IVIg may involve distinct pathways via FcRn or FcyRIIb receptors. Kaneko et al. highlighted the implication of the Fc $\alpha 2,6$-sialylated fraction of IVIg in a murine model of rheumatoid arthritis [17]. The production of sialylated IgGs with potentially enhanced specificity and efficacy may provide a safe alternative to IVIg and could also compensate for possible IVIg shortage. The ability to generate highly sialylated IgGs could also help in gaining a better understanding of their mechanism of action.

Several approaches are used to enhance the sialylation level of recombinant proteins produced in mammalian cell cultures, such as cell line engineering, bioprocess control, postproduction enrichment (such as lectin-affinity purification) and in vitro glycan remodelling $[27,40]$. In the field of mammalian cell engineering, a variety of approaches were developed. Antisense RNA and short interfering RNA were used in $\mathrm{CHO}$ cell lines to knock-down genes encoding for sialidases, leading to a significant increase in total amount of sialic acid in recombinant proteins $[39,41]$. Smaller but still significant improvement was achieved by the overexpression of CMP-sialic acid transporter, either alone [42] or in combination with CMP-sialic acid substrate [43]. Among the variety of approaches, the expression of the ST6Gal-I gene remains the only way to introduce $\alpha 2,6$-linked sialic acids on proteins in $\mathrm{CHO}$ cells.

The results obtained in this study were very similar to those of Jassal et al. on a monoclonal antibody of the IgG3 subtype [26]. The replacement of phenylalanine 246, equivalent to F243 in IgG3, by an alanine residue was necessary to reach significant sialylation levels, and the impact of this mutation was much stronger than that of human ST6Gal-I over-expression in both 293 and CHO cell lines. This confirms that IgG1 Fc structure strongly limits glycan accessibility to the sialyltransferases. The intact mass analysis profile of the mutant coexpressed with ST6Gal-I in CHO was dominated by the fully sialylated Mab, followed by tri-, di- and monosialylated forms.

Replacing the phenylalanine with an alanine residue decreases the hydrophobic environment that maintains the glycan buried in the Fc. Multiple mutation strategies are a 
powerful mean to promote glycosylation enhancement and Fc affinity for Fc $\gamma$ Rs. Computational modeling allows the design of antibodies with amino acid mutations that are not even exposed on the surface of the glycan pocket. However, the accumulation of mutations in a therapeutic Mab can have unpredictable side effects [44]. In the first place, mutation may compromise the affinity of the Mab for potential receptors involved in the anti-inflammatory mechanism. Also, non-natural Mab glycan structures (like sialylated triantennary glycans) may reduce their biological activity or even render them immunogenic. In a recent study, the CHO ST6Gal-I gene, present but not expressed, was cloned and stably expressed in these cells [45]. As a result, 70\% of the glycans of a stably expressed IgG1 and released by PNGaseF were sialylated. This striking result was thought to be conferred by a higher propensity of the CHO ST6Gal-I gene product for Fc glycan compared to its human or rat homologues, possibly due to some mutations in the substrate binding region. The use of this particular CHO ST6Gal-I gene may provide an interesting alternative strategy independent any IgG mutations to enhance sialylation of Mabs.

The production of highly sialylated IgGs in large quantities will probably be achieved by the simultaneous use of cell line engineering, Fc engineering, bioprocess control and downstream processing. Alternatively, optimizing in vitro glycan remodeling using ST6Gal-I and CMP-sialic acid may represent another cost-effective way to produce industrial amounts of sialylated IgGs [12]. From the perspective of obtaining a safe and reliable alternative to IVIg for anti-inflammatory applications, the production of sialylated recombinant Fc could be an attractive approach. However, developing these molecules as therapeutics awaits a better understanding of their mode of action.

\section{Author details}

Céline Raymond and Yves Durocher

Département de biochimie, Université de Montréal, Montréal, Canada

Céline Raymond, Erika Lattová, Hélène Perreault and Yves Durocher

MabNet NSERC Network for the Manufacturing of Single-Type Glycoform Monoclonal

Antibody

Anna Robotham, John Kelly and Yves Durocher

Human Health Therapeutic Portfolio of the National Research Council Canada, Montreal and

Ottawa, Canada

Erika Lattová and Hélène Perreault

Chemistry Department, University of Manitoba, Winnipeg, Canada

\section{Acknowledgement}

We thank Louis Bisson for protein-A HPLC quantifications. 


\section{References}

[1] Kozlowski, S. and P. Swann, Current and future issues in the manufacturing and development of monoclonal antibodies. Advanced drug delivery reviews, 2006. 58: p. 707-22.

[2] Munro, T.P., et al., Bridging the gap: facilities and technologies for development of early stage therapeutic mAb candidates. mAbs, 2011. 3: p. 440-52.

[3] Carter, P.J., Introduction to current and future protein therapeutics: a protein engineering perspective. Experimental cell research, 2011. 317: p. 1261-9.

[4] Reichert, J., M, Marketed therapeutic antibodies compendium. Mabs, 2012. 4(3): p. 413415.

[5] Lund, J., et al., Oligosaccharide-protein interactions in IgG can modulate recognition by Fc gamma receptors. FASEB J, 1995. 9(1): p. 115-9.

[6] Raju, T.S., Terminal sugars of Fc glycans influence antibody effector functions of IgGs. Curr Opin Immunol, 2008. 20(4): p. 471-8.

[7] Mizuochi, T., et al., Structural and numerical variations of the carbohydrate moiety of immunoglobulin G. The Journal of Immunology, 1982. 129: p. 2016-2020.

[8] Wormald, M.R., et al., Variations in oligosaccharide-protein interactions in immunoglobulin $\mathrm{G}$ determine the site-specific glycosylation profiles and modulate the dynamic motion of the Fc oligosaccharides. Biochemistry, 1997. 36: p. 1370-80.

[9] Deisenhofer, J., Crystallographic refinement and atomic models of a human Fc fragment and its complex with fragment B of protein A from Staphylococcus aureus at 2.9- and 2.8-A resolution. Biochemistry, 1981. 20: p. 2361-2370.

[10] Jefferis, R., et al., A comparative study of the N-linked oligosaccharide structures of human IgG subclass proteins. 1990. 268: p. 529-537.

[11] Krapp, S., et al., Structural analysis of human IgG-Fc glycoforms reveals a correlation between glycosylation and structural integrity. J Mol Biol, 2003. 325(5): p. 979-989.

[12] Barb, A.W., E.K. Brady, and J.H. Prestegard, Branch-specific sialylation of IgG-Fc glycans by ST6Gal-I. Biochemistry, 2009. 48(41): p. 9705-7.

[13] Morell, G., G. Gregoriadis, and I.H. Scheinberg, The Role of Sialic Acid in Determining Glycoproteins in the Circulation. The Journal of biological chemistry, 1971. 246: p. 14611467.

[14] Park, E.I., et al., The asialoglycoprotein receptor clears glycoconjugates terminating with sialic acid alpha 2,6GalNAc. Proc Natl Acad Sci U S A, 2005. 102(47): p. 17125-9.

[15] Unverzagt, C., et al., Structure-activity profiles of complex biantennary glycans with core fucosylation and with/without additional alpha 2,3/alpha 2,6 sialylation: synthesis of neoglycoproteins and their properties in lectin assays, cell binding, and organ uptake. J Med Chem, 2002. 45(2): p. 478-91. 
[16] Millward, T., et al., Effect of constant and variable domain glycosylation on pharmacokinetics of therapeutic antibodies in mice. Biologicals : journal of the International Association of Biological Standardization, 2008. 36: p. 41-7.

[17] Kaneko, Y., F. Nimmerjahn, and J.V. Ravetch, Anti-inflammatory activity of immunoglobulin G resulting from Fc sialylation. Science, 2006. 313(5787): p. 670673.

[18] Samuelsson, a., T.L. Towers, and J.V. Ravetch, Anti-inflammatory activity of IVIG mediated through the inhibitory Fc receptor. Science (New York, N.Y.), 2001. 291: p. 484-6.

[19] Anthony, R.M., et al., Identification of a receptor required for the anti-inflammatory activity of IVIG. Proc Natl Acad Sci U S A, 2008. 105(50): p. 19571-8.

[20] Siragam, V., et al., Intravenous immunoglobulin ameliorates ITP via activating Fc gamma receptors on dendritic cells. Nature Medicine, 2006. 12: p. 688-692.

[21] Crow, A.R., D. Brinc, and A.H. Lazarus, New insight into the mechanism of action of IVIg: the role of dendritic cells. Journal of thrombosis and haemostasis, 2009. 7 Suppl 1: p. 245-248.

[22] van de Geijn, F.E., et al., Immunoglobulin G galactosylation and sialylation are associated with pregnancy-induced improvement of rheumatoid arthritis and the postpartum flare: results from a large prospective cohort study. Arthritis research \& therapy, 2009. 11: p. R193.

[23] Patel, D.a., et al., Neonatal Fc receptor blockade by Fc engineering ameliorates arthritis in a murine model. Journal of immunology, 2011. 187: p. 1015-22.

[24] Ishii, N., et al., High-dose intravenous immunoglobulin (IVIG) therapy in autoimmune skin blistering diseases. Clinical reviews in allergy \& immunology, 2010. 38(2-3): p. 18695.

[25] Siberil, S., et al., Intravenous immunoglobulins in autoimmune and inflammatory diseases: a mechanistic perspective. Annals of the New York Academy of Sciences, 2007. 1110: p. 497-506.

[26] Jassal, R., et al., Sialylation of human IgG-Fc carbohydrate by transfected rat alpha2,6sialyltransferase. Biochem Biophys Res Commun, 2001. 286(2): p. 243-9.

[27] Durocher, Y. and M. Butler, Expression systems for therapeutic glycoprotein production. Curr Opin Biotechnol, 2009. 20(6): p. 700-7.

[28] Pham, P.L., A. Kamen, and Y. Durocher, Large-scale transfection of mammalian cells for the fast production of recombinant protein. Mol Biotechnol, 2006. 34(2): p. 225-37.

[29] Geisse, S. and C. Fux, Recombinant protein production by transient gene transfer into Mammalian cells. Methods in enzymology, 2009. 463: p. 223-38.

[30] Rajendra, Y., et al., A simple high-yielding process for transient gene expression in CHO cells. Journal of biotechnology, 2011. 153(1-2): p. 22-6.

[31] Tom, R., L. Bisson, and Y. Durocher, Culture of HEK293-EBNA1 Cells for Production of Recombinant Proteins. Cold Spring Harbor Protocols, 2008. 3(4): p. pdb.prot4976. 
[32] Shi, C., et al., Purification and Characterization of a Recombinant G-Protein-Coupled Receptor, Saccharomyces cerevisiae Step 2p, Transiently Expressed in HEK293 EBNA1 Cells. Biochemistry, 2005. 44: p. 15705-15714.

[33] Zhang, J., et al., Transient expression and purification of chimeric heavy chain antibodies. Protein Expr Purif, 2009. 65(1): p. 77-82.

[34] Loignon, M., et al., Stable high volumetric production of glycosylated human recombinant IFNalpha2b in HEK293 cells. BMC Biotechnol, 2008. 8: p. 65.

[35] Durocher, Y., S. Perret, and A. Kamen, High-level and high-throughput recombinant protein production by transient transfection of suspension-growing human 293-EBNA1 cells. Nucleic Acids Res., 2002. 30(2): p. E9.

[36] Lattová, E., O. Krokhin, and H. Perreault, Matrix-assisted laser desorption/ionization tandem mass spectrometry and post-source decay fragmentation study of phenylhydrazones of N-linked oligosaccharides from ovalbumin. J. Am. Chem. Soc. Mass Spectrom., 2004. 15: p.725-735.

[37] Yang, M. and M. Butler, Effects of ammonia and glucosamine on the heterogeneity of erythropoietin glycoforms. Biotechnol.Prog., 2002. 18(1): p. 129-138.

[38] Gawlitzek, M., et al., Ammonium alters N-glycan structures of recombinant TNFRIgG: degradative versus biosynthetic mechanisms. Biotechnol.Bioeng., 2000. 68(6): p. 637-646.

[39] Ferrari, J., et al., Chinese hamster ovary cells with constitutively expressed sialidase antisense RNA produce recombinant DNase in batch culture with increased sialic acid. Biotechnology and Bioengineering, 1998. 60: p. 589-595.

[40] Raju, T.S., et al., Glycoengineering of therapeutic glycoproteins: in vitro galactosylation and sialylation of glycoproteins with terminal N-acetylglucosamine and galactose residues. Biochemistry, 2001. 40(30): p. 8868-76.

[41] Ngantung, F.A., et al., RNA interference of sialidase improves glycoprotein sialic acid content consistency. Biotechnology and Bioengineering, 2006. 95(1): p. 106-19.

[42] Wong, N.S., M.G. Yap, and D.I. Wang, Enhancing recombinant glycoprotein sialylation through CMP-sialic acid transporter over expression in Chinese hamster ovary cells. Biotechnol.Bioeng., 2006. 93(5): p. 1005-1016.

[43] Jeong, Y.T., et al., Enhanced sialylation of recombinant erythropoietin in genetically engineered Chinese-hamster ovary cells. Biotechnol Appl Biochem, 2009. 52(Pt 4): p. 283-91.

[44] Kaneko, E. and R. Niwa, Optimizing therapeutic antibody function: progress with Fc domain engineering. BioDrugs : clinical immunotherapeutics, biopharmaceuticals and gene therapy, 2011. 25: p. 1-11.

[45] Onitsuka, M., et al., Enhancement of sialylation on humanized IgG-like bispecific antibody by overexpression of $\alpha 2,6$-sialyltransferase derived from Chinese hamster ovary cells. Applied microbiology and biotechnology, 2012. 94: p. 69-80.

[46] Raymond, C., et al., A simplified polyethylenimine-mediated transfection process for large-scale and high-throughput applications. Methods, 2011. 55(1): p.44-51. 
[47] Ciucanu, I., and F. Kerek, A simple method for the permethylation of carbohydrates. Carbohydr. Res. 1984; 131: p. 209-217.

[48] Schlaeger, E., and K. Christensen, Transient gene expression in mammalian cells grown in serum-free suspension culture. Cytotechnology, 1999, 30(1-3): p.71-83. 